\title{
Ways to Improve Medication Adherence in Chronic Disease Patients - A Review
}

\author{
Juhi Singh ${ }^{1}$, Md Shamshir Alam², Anuj Malik ${ }^{3}$, Shubham Singh Tyagi ${ }^{4}$, \\ Mohd Tousib ${ }^{5}$, Nawazish Alam ${ }^{6}$, Arinjay Jain ${ }^{7}$, Paluck ${ }^{8}$
}
1,2,3 Department of Pharmacy, MM College of Pharmacy, Maharishi Markandeshwar (Deemed to Be university), Mullana, India, ${ }^{4}$ Department of Pharmacy, College of Pharmacy, Teerthanker Mahaveer University, NH24, Moradabad, Uttar Pradesh, India. 5, 7,8 Department of Pharmacy, College of Pharmacy, Teerthanker Mahaveer University, NH24, Moradabad, Uttar Pradesh, India, ${ }^{6}$ Department of Clinical Pharmacy, College of Pharmacy, Jazan University, Jazan, P. O. Box 114, Kingdom of Saudi Arabia.

\section{ABSTRACT}

Adherence has been defined as the "voluntary, active, and collaborative involvement of the patients in mutually acceptable courses of behaviour to produce desired therapeutic effects". Medication adherence generally illustrates the term as to whether the patients take their medicines as per prescription instruction and either they keep on to take a prescribed medication.

Medication adherence performance has thus been classified into two head conceptions, namely, adherence and persistence. Whilst theoretically similar, adherence refers to concentration of drug utilization for the duration of the ongoing treatment, whereas persistence refers to the general interval of drug therapy. Improving prescription adherence may impact the well-being of the populace than the revelation of any new treatment.

Indian patients are not adherent to their medication half the time, mainly due to lack of proper education and patient counselling. Albeit most doctors do not accept adherence basically because of the absence of access or neglect, and no adherence can frequently be a purposeful decision made by the Indian patients. Persistent covering of their medicine taking conduct is regularly persuaded by feelings with respect to both supplier and the patient, prompting possible desperate outcomes. On time medicine taking behaviour of the patients have great impact on the health of people than the need for new treatment option.

\section{KEY WORDS}

Medication Adherence, Drug Therapy, Prescription, Wellbeing, Compliance, Chronic Disease
Corresponding Author: Dr. Md Shamshir Alam, Department of Pharmacy Practice, MM College of Pharmacy, Maharishi Markandeshwar (Deemed to Be University) Mullana, Ambala - 133207, India,

E-mail: shamshir_pharma@yahoo.com

DOI: $10.14260 / j e m d s / 2021 / 644$

How to Cite This Article:

Singh J, Alam MS, Malik A, et al. Ways to improve medication adherence in chronic disease patients - a review.J Evolution Med Dent Sci 2021;10(36):3171-3177, DOI: 10.14260/jemds/2021/644

Submission 11-05-2021,

Peer Review 27-07-2021,

Acceptance 05-08-2021,

Published 06-09-2021.

Copyright (C) 2021 Juhi Singh et al. This is an open access article distributed under Creative Commons Attribution License [Attribution 4.0 International (CC BY 4.0)] 


\section{BACKGROUND}

Patient adherence or compliance means correctly following of medical advice by the patients. Usually the patients take medication on his / her own called medication compliance, however the term adherence may also refer to the use of surgical appliances, like compression stocking, chronic wound care, self-directed physiotherapy exercises, or attending consoling or other therapy courses. ${ }^{1}$ World Health Organization (WHO) describes medication adherence as "the degree to which the individual's behaviour corresponds with the agreement to any advice or recommendations from a health care professional who provides health care. ${ }^{2}$ Patients with chronic disease conditions like asthma, chronic obstructive pulmonary disease, diabetes, and cardiovascular disease often require emergency attention and hospitalization sometimes due to non-adherence to the therapy, resulting in adverse outcome and higher treatment costs. Impoverished medication adherence is considered as a paramount public health challenge in health care system particularly in noncommunicable chronic disorders that require lifelong medication therapy. 2,3 According to the WHO estimation, almost half of the patients suffering from chronic diseases are non-adherent to their medication therapy. ${ }^{2} \mathrm{~A}$ study conducted in an out patient department at a university hospital in New Delhi, documented poor medication adherence in diabetes patients. ${ }^{4}$ Low rates of medication adherence in diabetic ${ }^{5}$ and hypertensive patients ${ }^{6}$ have also been documented in two different community - based studies in South Indian states.

Medication adherence is categorised into two main concepts that are adherence and persistence. Both the concepts are theoretically similar but adherence is the attentiveness of drug used for the time of the duration of therapy, while persistence refers to overall duration of medication therapy. ${ }^{7}$ It incorporates the inception of the treatment, usage of the endorsed system, and cessation of the pharmacotherapy. A few investigations characterized adherence as one or the other essential or optional tool. It doesn't just influence the clinical result yet additionally influence the monetary result of the wellbeing framework. Regularly, consistence is additionally utilized and the two can be utilized reciprocally in examination and clinical practice. It portrays "the degree to which the patients' conduct (counting prescription taking) harmonizes with clinical or medical care exhortation", yet its significance has become more negative in regard to patient's practices, since it infers patient's lack of involvement, consequently, in this paper, adherence will basically be utilized. Be that as it may, as indicated by Steiner and Earnest, the two terms present issues as to depict prescription taking practices since they expand the doctor's command over the drug cycle. ${ }^{9}$

It is evident from the literature that suboptimal medication adherence precludes the expected therapeutic outcome and hence enhances the risk of hospitalizations and adverse health consequences in patients that ultimately lead to additional economic burden to them. It is thus recognized that imparting patient education and utilizing medication adherence techniques and tools could be helpful for the betterment of general population health and hence lower healthcare costs. In this current review, we encompass various methods to enhance medication adherence which could be utilized by health care providers operating in health settings for improving patient medication adherence.

\section{METHODS}

The current review was conducted with the published articles available through online mode. The literature was searched with published sources from PubMed and Google Scholar search engines. All relevant articles published in English from 1978 to 2021, were retrieved, checked for suitability, and included in this review. The keywords used included patient adherence, medication adherence, patient compliance, factors influencing medication adherence and medication therapy adherence method. The barriers and challenges in continuing good therapeutic adherence and causes for poor adherence were identified from these studies.

The full articles were obtained by means of online resources. All the selected articles were thoroughly studied and the required data were extracted out which was transferred into computer generated sheet, evaluated and compiled in the form of present review.

\section{PROBLEMS WITH NON-ADHERENCE}

Therapeutic compliance is the most important clinical issue from the different health care provider's perspectives. Mainly there are two reasons, firstly the non-compliance could be the huge effect on the treatment of the patients care outcome and direct medical consequences. ${ }^{10}$ Also non-compliance is directly associated with the poor treatment outcome in several diseases and conditions. Like in hypertension non-compliance is the major reason for the uncontrollable increase in the blood pressure that can furthermore increase the risk of stroke, heart attack, myocardial infarction, and renal impairment. Several types of non-compliance are prevalent in the health practice. Non-compliance is commonly encountered in the clinical practice or in medical practice on a daily basis. For treatment modalities like exercise or diet, the accepted word compliance is either not defined uniformly in various studies conducted in diverse conditions or it appears to follow any universally accepted criterion to describe good or acceptable compliance. ${ }^{11}$

Non-compliance during disease management can create problems that not only has direct impact but also has indirect effect. The biggest problems associated with non-compliance are the development of resistance to microorganisms. Further, it has been observed that the majority of all patients who had poor compliance with the prescribed therapy ultimately stopped all the medications completely and hence were deprived of the perceived benefit of the suggested therapy. Non-compliance towards the therapy not only resulted in decline of clinical outcome but also lead to increase in financial burden to the society. ${ }^{12}$ For example, therapeutic noncompliance has been associated with the increase in the urgent care visits, also increase in hospitalization and higher treatment cost. Non-compliance also has negative effect on the patient's quality of life.

Sometimes when drug therapy has no effect as a consequence of undetected, unreported therapeutic non- 
compliance then doctor or health care personnels modify the regimen, which may raise the cost or complexity of the treatment regimen and hence increases extra unnecessary burden on healthcare cost of the patients. Non-adherence with the therapy reflects intentional as well as unintentional behaviour of the patients. Whatever be the cause of nonadherence; factors like medicine cost, lack of understanding, adverse effect, forgetfulness or not taking medicine can constitute the outcome to serious safety concerns. Further prescription medicine cost is the greatest growing component of the healthcare costs, for this reason from both the perspectives of achieving desirable clinical and economic outcomes, the negative aspects of therapeutic non-compliance need to be take into account on priority basis and minimize its effects to achieve the desired goal. ${ }^{13}$

\section{BENEFITS OF MEDICATION ADHERENCE}

Adherence is the most important thing because medication adherence defines to the degree or coverage of patients that comply with provider's suggested medication plan. Patient compliance is assumed when patients go along with the directions in terms of time, drug dose, and frequency of all their medicines. In reality head-to-head talk about medication adherence happens during patient's visit or hospital discharge time, however, the important point is that once the patient leaves the doctor's office or clinic, the patient itself has to take responsibility of his / her medication therapy. ${ }^{14}$

Taking medicines regularly for as long as prescribed by the prescriber, at the right time and dose, and according to instructions, could help feel well and stay well. Regular practicing medication adherence assures the maximum beneficial impact of the medicines taken. It could also minimize the risk associated with the drug therapy. ${ }^{11}$

Medication adherence reduces the cost of illness as the patient takes medicine (s) according to instructions and complies or adheres with the prescribed medication regimen. It would help patients feel good and heal quickly that would reduce the direct and indirect cost burden of the medication on the patient; ultimately reduce the overall cost of care. Also, medication adherence reduces the adverse outcomes. It increases the quality of life of the patient and would also be beneficial for the clinicians because sometimes variety of data sources are available and medication adherence measures would be deciding factors for particular patients who are not compliant with the therapy due to various reasons. ${ }^{3}$ Medication adherence is crucial for desired patient outcome as drugs do not show their therapeutic effects in patients who don't take medicines.

\section{FACTORS INFLUENCING} MEDICATION ADHERENCE

Notwithstanding the grouping of adherence measures as emotional and level-headed, numerous different investigations named them as direct and indirect. Thus, the prediction of medication adherence is difficult since the situation is different for each patient. ${ }^{15}$ Based on scientific research the factors influencing adherence or any healthrelated behaviours are summarized into 3 categories:

\section{Predisposing Factors ${ }^{16}$}

These factors include demographic factors of the patients, also their knowledge, attitudes, perception about illness, its severity, cause, prevention, and types of treatment regimen. The health belief model developed in 1974 predicted adherence or other health related behaviour changes in terms of certain belief patterns. The sequence of belief event under this model, which needs to occur if the patients are to be adherent, is as follows:

- The patients should believe that their health is in danger.

- The patient should identify the potential of the condition in terms of symptoms, time lost from work, economic difficulty, etc.

- After evaluating the conditions, the patients should believe that benefits from treatment compensate the costs.

- The patients should feel the need to adhere to the medication.

\section{Enabling Factors ${ }^{17}$}

These factors are the skills and recourses required for adherence. The term skill refers to the patients' capability of embracing such behaviour that will contribute to adherence to the medication regimen and hence take appointment from the doctor to obtain a prescription order. The term resources refer to the arability and approachability of health care facility such as doctors, pharmacies, clinics and hospitals.

\section{Reinforcing Factors ${ }^{\mathbf{1 8}}$}

Member, peers, health care providers of the local community and society would be supportive enough for the patients and encourage them to take medicines as directed by the prescriber. These factors determine whether or not the patient's family assists in medication adherence. This support may be positive or negative and this depends on the attitude or behaviour of the people as some may be more influential while the others may be less.

\section{MONITORING OF PATIENTS MEDICATION ADHERENCE}

\section{Direct Methods}

It incorporates estimation of the medication, or its metabolite focus in body liquids, for example, blood or plasma assessment of the presence of an organic marker given with the medication and direct perception of patient's medicine taking conduct. These measures can be made arbitrarily or at explicit spans. Despite the fact that immediate measures are viewed as the most precise and can be utilized as an actual proof to demonstrate that the patient has taken the medicine. There are numerous disadvantages of this method with respect to their utilization. They essentially produce a Yes / No outcome without uncovering any example of the no adherence or their causes. Tests themselves can likewise be extremely 
meddlesome which may be filled as weight and uneasiness in patients. ${ }^{17}$

Also, medication digestion should be considered while thinking about utilizing these techniques. For example, hints of narcoleptic and mental prescriptions can be recognized in the blood even long subsequent to halting the drug. Since people shift in physiological state and metabolic rate, drug plasma levels additionally vary after different people take a similar portion of a similar medication. Also, the evaluation itself can be a troublesome for assessing medication adherence. For example, riboflavin which is a natural marker is essentially not suitable for quantitative identification. Furthermore, drug drug interaction and medication food interference can alter the measure's precision. In conclusion, these immediate strategies are by and large inadmissible for mental patients and those under multidrug therapies, particularly, when the patients are hospitalized. 18

\section{Indirect Methods}

Indirect adherence measurement techniques include medication refill logs, patient self-reports, clinical reaction, and drug refill records. But the actual problems with these methods are that they are not much reliable and laboratory tests indicate that these are overestimated adherences.

The Morisky Medication Adherence Scale (MMAS) is a standardised screening instrument used in a number of patient groups to assess non-adherence with the therapy.19 Numerous trials on a global scale of over 110 literation with over 80 translations have confirmed and substantiated the creditability of this method. ${ }^{20}$ The method uses a set of brief behavioural questions formulated in such a way to prevent yes saying, the stigma is often seen in patients with longer treatment. More precisely, the wording of the questions is rearranged in order to eliminate replies that appear to pursue these behaviours is the only phenomena. This employs the patient to respond to caring personnel about non-adherence in a clinician's spirit of full disclosure. The Morisky Medication Adherence Scale, alternatively referred to as Morisky - Scale (MMAS8), has proven to be a reliable guide for resolving problems of therapy adherence, such as failing to take drugs or without instructions to discontinue medications. When a patient's score is higher on the numerical value, it indicates more adherents with the therapy. ${ }^{21}$

\section{Other Methods}

There are many methods which can be used for measuring the adherence like questionnaires and structured interviews, therapeutic drug monitoring, electronic medication monitoring, pick-up and refill rates, medication event monitoring system, medical signals, smart phones, reminder systems, etc. 22

Questionnaires and Structured Interviews Self-directed questionnaires are regarded as a very reasonable, indirect, and well-organized techniques to assess patient adherence with the therapy. These methods can be very easily applicable in clinical practice and incurs low cost. But they may be problematic sometimes, particularly when the counsellor is not well prepared for scheduled counselling session.

\section{Therapeutic Drug Monitoring}

Therapeutic drug monitoring is the only direct objective measure of medication adherence. In this method drug concentration of the body fluids, serum and plasma of the patient is measured. This method is mostly used as an adherent tool to customize drug treatment to modify doses in the target drug concentration.

This method has advantages over others as in this method small quantity of blood volume is needed; therefore, it reduces the risk of transferring infections and pathogens. Also, specimens of other matrices can also be used for the estimation of medication adherence through this method. ${ }^{23}$

\section{Electronic Medication Monitoring}

The introduction of electronic monitoring system to evaluate drug therapy adherence has been an important adding to the existing adherence quantification technique. This device has been even regarded as the gold standard of medication adherence measurement.24 Oral medication monitoring consists of the electronic drug exposure monitor system.

\section{Medication Event Monitoring System}

Medication event monitoring system (MEMS) comprises of computer chip connected with the bottle cap, which can record the time as well as the date each time the pill bottle is opened. ${ }^{25}$ The MEMS has the capability to record both the drug dose as well as adherence with the therapy, thus has the advantage over other methods. The electronically recorded aperture intervals all the way through this system give healthcare providers insight into the medication taking habits of their patients. This system appears to improve drug therapy adherence to some extent, particularly when this technique is used for a short time. ${ }^{26}$

\section{Medical Signal}

It is an electronic pill container which is utilized in the management of medicine consumption by giving the real - time response on the patient's adherence. ${ }^{27}$

\section{Real Time Medication Monitoring System (RTMM)} Here the collection of the obtained data is done by sending them to a study database through a mobile telephone network. ${ }^{28}$ This system is equally simple for the patient and relatively easy to put in daily clinical practice. ${ }^{29}$

\section{The Real Time Wireless Electronic Adherence Monitor System}

This is a realistic and a suitable technique for measuring adherence in view of the opportunity it offers to intercede with adherence questions directly. This method has the disadvantage of its technological difficulties and price associated concerns. ${ }^{30}$ In recent times with the multifunctionality of electronic mobile devices, smart phones have been established to be helpful in the quantification and enhancement of medication adherence in the short - lived application. Reminder technique and short message facility text message can be adjusted with this track device. ${ }^{31}$ 


\section{Pick-Up and Refill Rates}

In pick-up and refill rates method pharmacy dispensing files are evaluated to measure patient medication adherence. The pick-up rates explain the number of picked up prescriptions as a percentage of the total advised doses. ${ }^{32}$ The refill rates are defined as the splitting up of the quantity of the days, the drugs have been advised by the total calendar days of that period, alienated by the total number of the days in that time period. ${ }^{33}$

\section{Electronic Medication Dispensing System}

This enhances drug therapy adherence by dispensing prepackaged medications in good time with reminder cautions while tracking medications and changes to symptoms perceived by the users. In a pilot randomized controlled trial, the researchers documented that the MDS enhanced the perceived health position of users over time, while the control groups did not recognize improvements in their health. The average medication adherence documented by the use of MDS over the six-month study duration was $98.4 \%{ }^{34}$ Similar study associated to medication dispensing technology reported participants' average adherence range which was from 97 to $98 \%{ }^{35}$ The MDS could be a successful, long-term solution to medication non-adherence in older adults with chronic disease conditions and taking different medications. ${ }^{36}$

\section{Barriers / Factors Leading to Non-Adherence}

Poor adherence to medication in both long term and shortterm duration circumstances enhance the risk of hospitalization, death, and cost of care. The part of the complexity in addressing non-adherence is its complication, as adherence could be influenced by several factors. The factors can be barriers to adherence and generally classified as either perceptual or practical. Drug formulation factors among the unambiguous formulation of the medications like size of the oral medication, size of the oral dosage form and difficulties associated with injections, were some of the recognized factors influencing adherence. ${ }^{37}$ From time to time some issues while taking the medication as advised and burden associated with the medication taking behaviour integrated items ranging from dosing frequency, shortage of medication requirements. Some of the specific restrictions with the medication intake like medicine with food, or at particular time of the day, impact on daily activities like personality, social life, work, indirect medication effects like side effects, medication monitoring on daily activities, occasionally on issues with remembering or forgetting doses because of busy schedules. Medication cost is also a great factor for the noncompliance; integrated with both direct and indirect cost relating to medication administrating like travelling expenses medication adherence may be medication refills. Occasionally social pressure like embarrassment in taking medication in front of other people and stigma related to utilization of medication can influence drug therapy adherence. 38

\section{Tips to Improve Medication Adherence ${ }^{39}$}

To improve patient adherence, physicians have to build positive physician patient relationship. Improving adherence has been a continuous cycle in the health care training. Like most practices, we requested patients to carry all their medicines to each office visit, so the observers could to obtain the medication supply. Other factor influencing

accommodate them at the point when they previously began a programme to keep patients out of the health-related problems and prevent the threat of readmissions. The medication adherence programme is conducted to analyse and see every patient's prescription medication taking behaviour. To find out if they experience difficulty in filling, taking, or managing the cost of their prescriptions, the following points are emphasized.

1. Discussion about results

2. Record it

3. Team up with patients

4. Think about the monetary weight to the patient

5. Evaluate well-being education

6. Lessen unpredictability

7. Catch up with patients.

\section{Role of Pharmacist in Medication Adherence}

Drug specialists have a significant role in improving prescription adherence in patients. They can affirm that patients are on the right medicines and are not taking any other medicines that may affect the viability of suggested treatment regimen. Drug therapy management can assist drug specialists in recognizing the risk of patients and help them to settle their prescription adherence issues. ${ }^{40}$ They can instruct patients about the correct use of medication on stipulated time, appropriate drug store applications to set medicine updates on their telephones. There are a few other clinical intercessions that drug specialists can execute to urge their patients to take the correct measurements on time, get top off on schedule and to manage symptoms of medications in emergency condition. ${ }^{41}$ The patient - pharmacist relationship may be improved by applying the following strategies ${ }^{42}$ :

1. The pharmacists should be friendly and approachable to the patients.

2. They should have very good communication skills.

3. The pharmacist should consider the physiological needs of the patients.

4. They should impart patient counselling and improve patients' education.

5. They should encourage every patient to discuss their concern without interruption or premature closing of the session.

6. They should extract patient's perception of illness and associated feelings and expectations.

7. They should utilize the method of active listing and show empathy to the patients.

8. They give their clear explanation to the patients.

9. They should check patients understanding level about the disease as well as the medicines.

10. They should discuss treatment plans with the patients.

\section{CONCLUSIONS}

In this article we understood that medical adherence is the degree to which the person's behaviour corresponds to stick with the direction of a healthcare giver. Ample proof shows that numerous patients with ongoing diseases experience issues not sticking to their recommended drug routine. Accepting that prescription, no adherence is the "shortcoming" of the patient is an ignorant and dangerous model that is best deserted. As the previous Surgeon General J Evolution Med Dent Sci / eISSN - 2278-4802, pISSN - 2278-4748 / Vol. 10 / Issue 36 / Sept. 06, 2021 
C Ever Koop reminded us, "Medications don't work in patients who don't take them". Thus, doctors should perceive that helpless drug adherence adds to problematic clinical conditions, especially considering the WHO explanation that expanding adherence may greatly affect patient wellbeing than any improvement in explicit clinical treatments. In essence adherence is an integral part of the medication therapy management that should be ensured in the patient management to maximize the desired therapeutic outcome.

Financial or other competing interests: None.

Disclosure forms provided by the authors are available with the full text of this article at jemds.com.

\section{REFERENCES}

[1] Apter AJ, Reisine ST, Affleck G, et al. Adherence with twice-daily dosing of inhaled steroids. Socioeconomic and health-belief differences. Am J Respir Crit Care Med 1998;157(6 Pt 1):1810-17.

[2] Sabate E. Adherence to long-term therapies: evidence for action. $1^{\text {st }}$ edn. Geneva, Switzerland: World Health Organization 2003.

[3] Vrijens B, Antoniou S, Burnier M, et al. Current situation of medication adherence in hypertension. Front Pharmacol 2017;8:100.

[4] Alam MS, Aqil M, Qadry SAS, et al. Utilization pattern of oral hypoglycemic agents for diabetes mellitus type 2 patients attending out-patient department at a university hospital in New Delhi. Pharmacology \& Pharmacy 2014;5(7):636-45.

[5] Sankar UV, Lipska K, Mini GK, et al. The adherence to medications in diabetic patients in rural Kerala, India. Asia Pac J Public Health 2015;27(2):NP513-23.

[6] Venkatachalam J, Abrahm SB, Singh Z, et al. Determinants of patient's adherence to hypertension medications in a rural population of Kancheepuram District in Tamil Nadu, South India. Indian J Community Med 2015;40(1):33-7.

[7] Bartlett EE, Grayson M, Barker R, et al. The effects of physician communications skills on patient satisfaction; recall and adherence. J Chronic Dis 1984;37(9-10):75564.

[8] Brown MT, Busell JK. Medication adherence: WHO Cares? Mayo Clin Proc 2011;86(4):304-14.

[9] Brown MT, Busell J, Dutta S, et al. Medication adherence: truth and consequences. Am J Med Sci 2016;351(4):38799.

[10] Bond WS, Hussar DA. Detection methods and strategies for improving medication compliance. Am J Hosp Pharm 1991;48(9):1978-88.

[11] Christensen DB. Drug-taking compliance: a review and synthesis. Health Serv Res 1978;13(2):171- 87

[12] Claxton AJ, Cramer J, Pierce C. A systematic review of the associations between dose regimens and medication compliance. Clin Ther 2001;23(8):1296-310.

[13] Cramer JA, Mattson RH, Prevey ML, et al. How often is medication taken as prescribed? A novel assessment technique. JAMA 1989;261(22):3273-7.

[14] Cramer JA, Scheyer RD, Mattson RH. Compliance declines between clinic visits. Arch Intern Med 1990;150(7):150910.
[15] Cramer JA. Enhancing patient compliance in the elderly. Role of packaging aids and monitoring. Drugs Aging 1998;12(1):7-15.

[16] CBO. Prescription drug coverage and Medicare's fiscal challenges. Congressional Budget Office 2003.

[17] Dhanireddy KK, Maniscalco J, Kirk AD. Is tolerance induction the answer to adolescent non-adherence? Pediatr Transplant 2005;9(3):357-63.

[18] DiMatteo MR. Patient adherence to pharmacotherapy: the importance of effective communication. Formulary 1995;30(10):596-605.

[19] Lam WY, Fresco P. Medication adherence measures: an overview. Biomed Res Int 2015;2015:217047.

[20] Dowse R, Ehlers M. Medicine labels incorporating pictograms: do they influence understanding and adherence? Patient Educ Couns 2005;58(1):63-70.

[21] Al-Qazaz HKh, Hassali MA, Shafie AA, et al. The eight-item morisky medication adherence scale MMAS: translation and validation of the Malaysian version. Diabetes Res Clin Pract 2010;90(2):216-21.

[22] Gonzalez J, Williams JW Jr, Noel PH, et al. Adherence to mental health treatment in a primary care clinic. J Am Board Fam Pract 2005;18(2):87-96.

[23] Gordis L. Conceptual and methodologic problem in measuring patient compliance. In: Haynes B, Taylor DW, Sackett DL, eds. Compliance in health care. Baltimore: John Hopkins University Press 1979:23-45.

[24] Jette AM, Rooks D, Lachman M, et al. Home-based resistance training: predictors of participation and adherence. Gerontologist 1998;38(4):412-21.

[25] Kyngas H, Rissanen M. Support as a crucial predictor of good compliance of adolescents with a chronic disease. J Clin Nurs 2001;10(6):767-74.

[26] Wetzels GE, Nelemans PJ, Schouten JS, et al. Electronic monitoring of adherence as a tool to improve blood pressure control. A randomized controlled trial. Am J Hypertens 2007;20(2):119-25.

[27] Murray MD, Darnell J, Weinberger M, et al. Factors contributing to medication noncompliance in elderly public housing tenants. Drug Intell Clin Pharm 1986;20(2):146-52.

[28] Neal RD, Hussain-Gambles M, Allgar VL, et al. Reasons for and consequences of missed appointments in general practice in the UK: questionnaire survey and prospective review of medical records. BMC Fam Pract 2005;6:47.

[29] Sluijs E, Van Dulmen S, Van Dijk L, et al. Patient adherence to medical treatment: a meta review. NIVEL 2006.

[30] Nichols-English G, Poirier S. Optimizing adherence to pharmaceutical care plans. J Am Pharm Assoc (Wash) 2000;40(4):475-85.

[31] Nikolaus T, Kruse W, Bach M, et al. Elderly patients' problems with medication. An in-hospital and follow-up study. Eur J Clin Pharmacol 1996;49(4):255-9.

[32] Okuno J, Yanagi H, Tomura S, et al. Compliance and medication knowledge among elderly Japanese homecare recipients. Eur J Clin Pharmacol 1999;55(2):145-9.

[33] Osterberg L, Blaschke T. Adherence to medication. N Engl J Med 2005;353(5):487-97.

[34] Arain MA, Ahmad A, Chiu V, et al. Medication adherence support of an in-home electronic medication dispensing system for individuals living with chronic conditions: a pilot randomized controlled trial. BMC Geriatr 2021;21(1):56. 
[35] Buckwalter KC, Wakefield BJ, Hanna B, et al. New technology for medication adherence: electronically managed medication dispensing system. J Gerontol Nurs 2004;30(7):5-8.

[36] Ahmad A, Chiu V, Arain MA. Users' perceptions of an inhome electronic medication dispensing system: a qualitative study. Med Devices Evid Res (Auckl) 2020;13:31-9.

[37] Eisen SA, Miller DK, Woodward RS, et al. The effect of prescribed daily dose frequency on patient medication compliance. Arch Intern Med 1990;150(9):1881-4.

[38] Feinstein AR. On white-coat effects and the electronic monitoring of compliance. Arch Intern Med 1990;150(7):1377-8.
[39] Tyagi S, Krishnaveni K. Book of Pharmacy Practice. Thakur Publication 2020:103-13.

[40] Ponnusankar S, Surulivelrajan M, Anandamoorthy N, et al. Assessment of impact of medication counseling on patients' medication knowledge and compliance in an outpatient clinic in South India. Patient Educ Couns 2004;54(1):55-60.

[41] Rosenstock IM, Strecher VJ, Becker MH. Social learning theory and the health belief model. Health Educ Q 1988;15(2):175-83.

[42] Roter DL, Hall JA. Why physician gender matters in shaping the physician-patient relationship. J Womens Health 1998;7(9):1093-7. 